\title{
Maturation of Phasic and Continuity Measures during Sleep in Preterm Neonates ${ }^{1}$
}

\author{
MARK S. SCHER, DORIS A. STEPPE, S. GEORGE DOKIANAKIS, AND \\ ROBERT D. GUTHRIE \\ Developmental Neurophysiology Laboratory, Magee-Womens Hospital, and Department of \\ Pediatrics, Children's Hospital of Pittsburgh, University of Pittsburgh School of Medicine, \\ Pittsburgh, Pennsylvania 15213
}

\begin{abstract}
ABS
Different physiologic measures during EEG sleep peri-
ods in preterm neonates are postulated to change with maturation and reflect functional brain development. Forty-three healthy preterm neonates received 3-h EEG sleep studies in an environmentally controlled setting. Postconceptional ages of neonates at each recording session ranged from 28 to $35 \mathrm{wk}$. Minute-by-minute analyses of EEG discontinuity, motility, arousals, and REM were performed. Eight phasic events and continuity measures of sleep were tabulated. Data were analyzed using Spearman rank order correlation coefficients. Increases in arousal numbers $(p<0.001)$ and durations $(p<0.001)$ were noted with age only during continuous periods of EEG activity (i.e. active sleep). REM also increased with corrected age during indeterminate or transitional sleep $(p<0.002)$ and decreased during quiet sleep $(p<0.01)$. Decreases in small body movements per minute $(p=0.02)$ and large body movements per minute $(p<0.001)$ occurred only during
\end{abstract}

The concept of state during early brain ontogenesis of the preterm infant is controversial. It is generally accepted that patterns representing sleep in preterm infants are highly variable (1) and less organized than patterns described for full-term infants $(2-4)$. Conventional wisdom dictates that organized sleep states do not appear before $31 \mathrm{wk}(1,2)$ and are not well established until 36 wk postconceptional age (1-6). Several researchers, however, have questioned this assumption based on studies of sleep in preterm infants. Rudimentary state differentiation in terms of motor, EEG discontinuity, and autonomic function have recently been described in early preterm infants $(4,7-18)$. Although four well-developed

Received February 8, 1993; accepted May 18, 1994.

Correspondence and reprint requests: Mark S. Scher, M.D., Developmental Neurophysiology Laboratory, Magee-Womens Hospital, 300 Halket St., Pittsburgh, PA 15213-3180.

Supported in part by Grants NS01110, NS26793, and M01RR00084, the Twenty-Five Club of Magee-Womens Hospital, and the Scaife Family Fund.

${ }^{1}$ Presented in part at the 7th International Child Neurology Meeting, Buenos Aires, Argentina, November 1992. discontinuous periods of EEG activity (i.e. quiet sleep). Sleep efficiency $(p<0.001)$, maintenance $(p<0.001)$, and latency $(p=0.01)$ also decreased with increasing postconceptional age. Cycle length between two segments of continuous EEG with an intervening period of EEG discontinuity also lengthened with maturation $(p<0.001)$. These findings are discussed in the context of previously reported differences in phasic and continuity measures noted between preterm and full-term infants at matched full-term postconceptional ages. Changes in phasic and continuity measures with increasing postconceptional ages reflect maturation of specific neuronal processes of the CNS within a rudimentary sleep cycle of the preterm neonate. (Pediatr Res 36: 732-737, 1994)
REM, rapid eye movement

sleep state segments of the full-term infant are not observed on the EEG recordings of preterm infants, specific physiologic measures may already have an established association during specific segments of the rudimentary EEG sleep cycle.

Studies of the maturational trends of polysomnographic measures in asymptomatic, presumably healthy neonates will assist in the documentation of normative data, which can then be applied to the evaluation of neonates at risk for CNS disorders. Changes in phasic and continuity measures with maturation during sleep from 28-35 wk postconceptional age were investigated in a group of 43 healthy preterm neonates who were assessed as neurodevelopmentally normal on follow-up assessment.

\section{METHODS}

Asymptomatic preterm neonates were prospectively recruited at $\leq 32 \mathrm{wk}$ estimated gestational age from the neonatal intensive care unit at Magee-Womens Hospital and studied between postconceptional ages 28 and $35 \mathrm{wk}$. 
No child had major medical illnesses, and all received at least one cranial ultrasound that documented no significant intracranial hemorrhage or other brain lesions. None required ventilatory assistance. Three $h$ of paperrecorded EEG polysomnographic studies were obtained between 2100 and $2400 \mathrm{~h}$ as part of an all-night sleep study. These 43 infants were assessed in our neurology follow-up clinic from 18 mo to $3 \mathrm{y}$ of age and were judged to be neurodevelopmentally normal, either by examination or parental reportage.

Twenty-one channels of EEG were recorded on a Nihon-Khoden (model 4221, Irvine, CA) EEG machine. Fourteen EEG channels, one electromyogram, two electrooculograms, two respiration, and one ECG channel were used. The time constant was $0.3 \mathrm{~s}$ with a paper speed of $15 \mathrm{~mm} / \mathrm{s}$ and sensitivity of $7 \mu \mathrm{V} / \mathrm{mm}$. One EEG technologist documented movements, behavioral changes, and artifacts during each recording, which began after a feeding and diaper change. Review of the maternal and neonatal medical records was carried out by a neonatal nurse. All infants received a neurodevelopmental assessment on the day of the recording (19).

Scores of sleep state, arousals, and motility were tabulated per minute, as previously described (20). Minuteby-minute scores of EEG state and arousal number and duration (in seconds) were tabulated by the electroencephalographer. Body movements and REM were tabulated per minute by the EEG technologist. Body movements per minute included five categories (i.e. total, face, head, small, and large). All body movements were noted within a given minute, regardless of whether multiple movement types were observed. No durations of these movements were recorded unless the movement was judged to accompany an arousal as defined below. Large body movements were identified if simultaneous movements involving multiple muscle groups in an axial or appendicular distribution were observed (e.g. startle or squirm). Small body movements were identified if only isolated limb movements were observed (e.g. head or arm). Head movements were distinguished from isolated facial movements such as grimacing or sucking.

REM have been previously defined (20) and were identified when synchronized out-of-phase pen deflections in the two electrooculographic channels of at least $20 \mathrm{mV}$ in amplitude were observed.

Intra- and interscorer reliability for sleep measures was greater than $90 \%$. These hand-scored measures were entered into the database; extracted files were analyzed by the SAS statistical package (SAS, Inc., Cary, NC).

State definitions included five EEG states based on the total seconds of quiescence per minute, which were similar to definitions by Connell et al. (9) and Eyre et al. (10) (Table 1). State 1 was characterized as completely continuous EEG background rhythms, and four degrees of EEG discontinuity, through state 5, were also defined (Fig. 1). Because minutes of sleep were classified by the total number of seconds of EEG quiescence or inactivity, comparisons between EEG and polygraphic measures
Table 1. Categories of state for preterm infants based on EEG discontinuity*

State 1: continuous EEG (active sleep)

State 2: quiescent periods $\leq 12 \mathrm{~s}$ (active sleep)

State 3: quiescent periods $\leq 23 \mathrm{~s}$ (quiet sleep)

State 4: quiescent periods $\leq 33 \mathrm{~s}$ (quiet sleep)

State 5: quiescent periods $\leq 47 \mathrm{~s}$ (quiet sleep)

Transitional sleep: minutes of EEG recording during which periods of quiescence could not be identified because of artifact (e.g. movements, manipulation of the infant)

* Total $=$ seconds of quiescence per minute.

were not initially used for state identification. However, certain minutes contained behavioral arousals that would not be included as strictly continuous or discontinuous EEG segments. Minutes of indeterminate or transitional sleep were therefore scored if more than $30 \mathrm{~s}$ of that particular minute could still be assigned an EEG discontinuity score, although an obvious behavioral arousal was observed. For instances when artifact obscured the interpretation of EEG background activity for more than 30 $s$ of that minute, the minute was not included in the analyses. Fewer minutes of transitional sleep were then available for analysis than states 1 through 5 .

A number of polysomnographic measures were defined. Cycle length was calculated as the number of minutes of sleep between two consecutive segments of at least 3 min of an identical EEG sleep state separated by a dissimilar EEG state (e.g. a continuous 3-min segment of state 2 to the next 3-min segment of state 2). Cycle length based on state 2 was chosen because this EEG sleep state was defined as the least amount of seconds to classify quiet sleep. Arousals were defined as electrographic reactivity with sudden attenuation or diminution of EEG background rhythms, with or without behavioral responses or movements. Arousal events were distinguished from periods of EEG discontinuity in that movements or electromyogram artifact were not present during the latter segment of the sleep cycle. These were described as both numbers per minute and duration in seconds per minute.

"Lights out" was the time at which the sleep study began and from which sleep latency, efficiency, and maintenance were defined. Sleep onset was uniformly defined as the first full minute of sleep after lights out. Sleep latency was the amount of time from lights out to the first full minute of sleep. Sleep efficiency was the percent of total recording time spent asleep, and sleep maintenance was the percent of minutes of sleep after the first minute of sleep.

Histograms were used as an exploratory tool to assess the distributions of each variable. Because of nonnormal distributions, Spearman rank order correlations were then performed on each variable at increasing postconceptional ages.

\section{RESULTS}

Forty-three EEG sleep studies, one per neonate, were obtained from neonates ranging from 28 to $35 \mathrm{wk}$ post- 


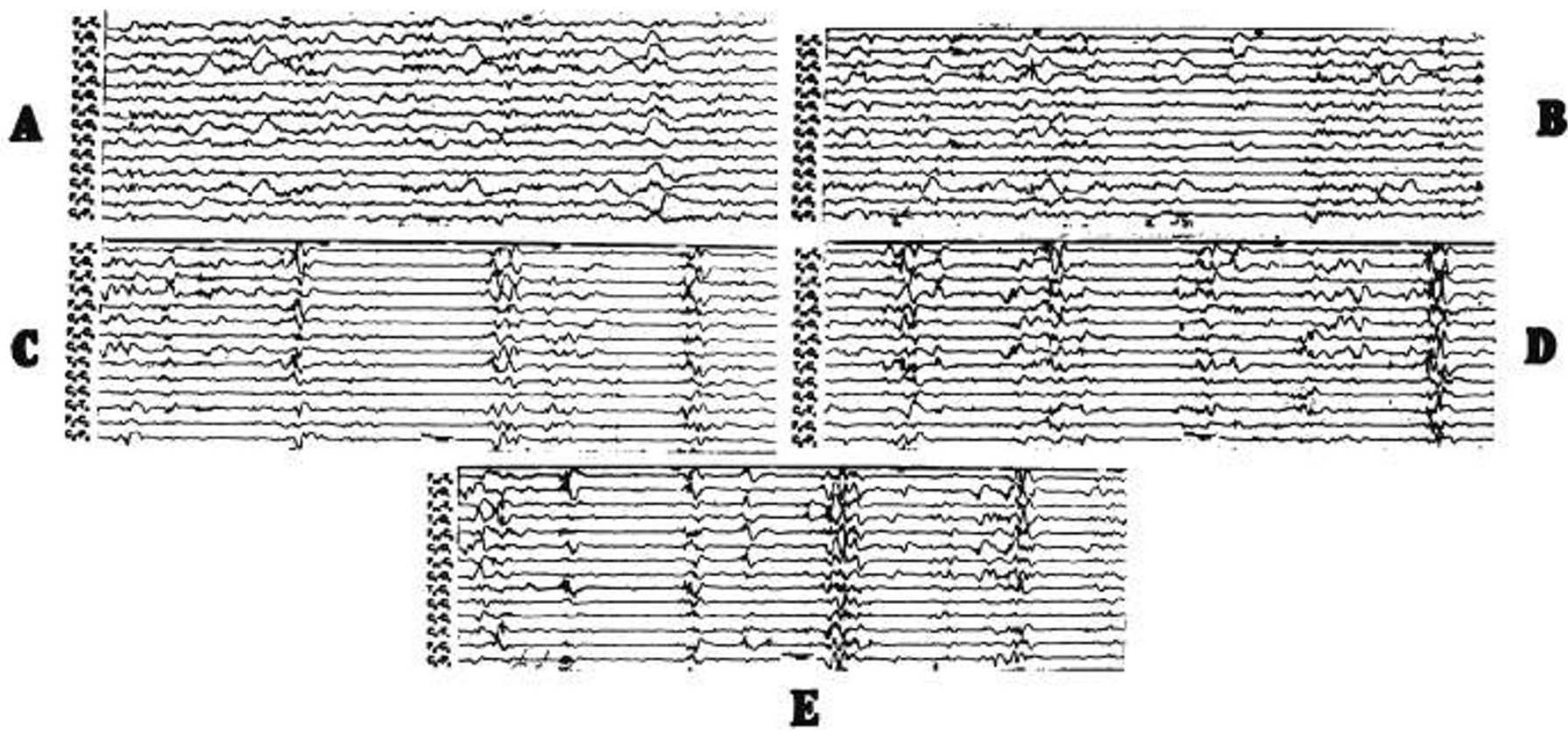

Figure 1. One-min epochs of EEG representing state 1 (panel $A$ ) through state 5 (panel E). States 2 through 5 demonstrate increasing EEG discontinuity. Polygraphic channels not shown.

conceptional age as listed in Table 2. Descriptive statistics for all sleep measures are given in Tables 3 through 5. Tables 6 and 7 give correlation coefficient results of these sleep measures.

Arousals. Postconceptional age was found to have a significant positive correlation with the number of arousals as well as the duration of arousals per minute. This, however, was only significant for continuous EEG states (i.e. states 1 and 2$) ; r$ values were 0.69 and 0.68 , respectively $(p<0.001)$.

REM. Significant positive correlations with maturation were seen with respect to the number of REM per minute during transitional or indeterminate segments of EEG sleep (i.e. $r$ of $0.54 ; p<0.002$ ). Transitional sleep segments occurred in only nine of the 21 neonates younger than 33 wk postconceptional age. Significant negative correlations for REM per minute with age were noted, however, during discontinuous sleep (i.e. $r=-0.39, p<0.01$ ).

Cycle length. Cycle length based on state 2 of EEG discontinuity showed a positive correlation with increasing postconceptional age, with an $r$ of $0.59(p<0.001)$. Cycle lengths based on the other four states showed no significant trends with maturation.

Sleep efficiency, maintenance, and latency measures. Negative correlations were noted with respect to sleep efficiency and maintenance, both with decreasing values as the preterm infant matured to $35 \mathrm{wk}$ postconceptional ages; $r$ values for both measures were -0.72 with significance levels of $<0.001$.

Table 2. Postconceptional ages of 43 neonates

\begin{tabular}{lllll}
\hline Postconceptional age (wk) & $28-29$ & $30-31$ & $32-33$ & $34-35$ \\
Subjects & 3 & 13 & 15 & 12 \\
Range of postnatal age (wk) & $1.6-3.0$ & $0.7-3.6$ & $1.7-4.6$ & $1.3-5.0$ \\
\hline
\end{tabular}

Sleep latency also showed a negative correlation, only with continuous sleep (i.e. state 1$)$, with an $r$ of $-0.42(p$ $<0.01)$.

Table 3. Descriptive statistics for postconceptional ages 28 to $35 w k$

\begin{tabular}{lrrc}
\hline \multicolumn{1}{c}{ Variable } & Mean & \multicolumn{1}{c}{ SD } & Range \\
\hline Continuous sleep $(n=43)$ & & & \\
Postconceptional age (wk) & 32.27 & 1.88 & $28.00-35.00$ \\
Arousal duration $(\mathrm{s} / \mathrm{min})$ & 2.19 & 2.08 & $0-6.65$ \\
Arousal number/min & 0.15 & 0.15 & $0-0.48$ \\
REM/min & 2.04 & 1.61 & $0.11-7.80$ \\
Head movements/min & 0.11 & 0.08 & $0-0.45$ \\
Face movements/min & 0.64 & 0.29 & $0.08-1.24$ \\
Small body movements/min & 0.16 & 0.15 & $0-0.69$ \\
Large body movements/min & 0.28 & 0.12 & $0.04-0.63$ \\
Total body movements/min & 1.18 & 0.42 & $0.40-2.27$ \\
Discontinuous sleep $(n=43)$ & & & \\
Arousal duration $(\mathrm{s} / \mathrm{min})$ & 0.26 & 0.47 & $0-2.31$ \\
Arousal number/min & 0.02 & 0.03 & $0-0.14$ \\
REM/min & 0.03 & 0.09 & $0-0.41$ \\
Head movements/min & 0.04 & 0.05 & $0-0.17$ \\
Face movements/min & 0.40 & 0.36 & $0-1.44$ \\
Small body movements/min & 0.09 & 0.11 & $0-0.43$ \\
Large body movements/min & 0.18 & 0.20 & $0-1.01$ \\
Total body movements/min & 0.71 & 0.50 & $0-1.93$ \\
Indeterminate sleep $(n=31)$ & & & \\
Arousal duration $(\mathrm{s} / \mathrm{min})$ & 24.78 & 11.69 & $0-46.67$ \\
Arousal number/min & 0.68 & 0.35 & $0-1.14$ \\
REM/min & 0.54 & 0.69 & $0-2.77$ \\
Head movements/min & 0.21 & 0.24 & $0-1.00$ \\
Face movements/min & 0.42 & 0.40 & $0-1.71$ \\
Small body movements/min & 0.13 & 0.20 & $0-1.00$ \\
Large body movements/min & 0.89 & 0.50 & $0-2.00$ \\
Total body movements/min & 1.64 & 0.79 & $0-4.00$ \\
All sleep states & & & \\
Latency to state 1 (min) $(n=39)$ & 19.10 & 20.11 & $0-63.00$ \\
Efficiency $n=43)$ & 0.92 & 0.10 & $0.71-1.00$ \\
Maintenance $(n=43)$ & 0.92 & 0.10 & $0.71-1.00$ \\
Cycle length (min) $(n=39)$ & 24.00 & 21.46 & $4.00-92.00$ \\
\hline & & & \\
& & & \\
& & &
\end{tabular}


Table 4. Descriptive statistics for postconceptional ages 28 to $31 \mathrm{wk}$

\begin{tabular}{|c|c|c|c|}
\hline Variable & Mean & $\mathrm{SD}$ & Range \\
\hline \multicolumn{4}{|l|}{ Continuous sleep $(n=16)$} \\
\hline Postconceptional age (wk) & 30.19 & 0.91 & $28.00-31.00$ \\
\hline Arousal duration (s/min) & 0.53 & 0.79 & $0-2.40$ \\
\hline Arousal number/min & 0.04 & 0.05 & $0-0.13$ \\
\hline $\mathrm{REM} / \mathrm{min}$ & 1.97 & 1.81 & $0.11-7.80$ \\
\hline Head movements/min & 0.07 & 0.06 & $0-0.17$ \\
\hline Face movements/min & 0.55 & 0.23 & $0.24-1.13$ \\
\hline Small body movements/min & 0.17 & 0.14 & $0-0.45$ \\
\hline Large body movements/min & 0.27 & 0.14 & $0.04-0.63$ \\
\hline Total body movements/min & 1.07 & 0.36 & $0.40-1.73$ \\
\hline \multicolumn{4}{|l|}{ Discontinuous sleep $(n=16)$} \\
\hline Arousal duration (s/min) & 0.20 & 0.58 & $0-2.31$ \\
\hline Arousal number/min & 0.01 & 0.04 & $0-0.14$ \\
\hline $\mathrm{REM} / \mathrm{min}$ & 0.08 & 0.13 & $0-0.41$ \\
\hline Head movements/min & 0.03 & 0.03 & $0-0.08$ \\
\hline Face movements/min & 0.30 & 0.16 & $0.07 \sim 0.70$ \\
\hline Small body movements/min & 0.14 & 0.15 & $0-0.43$ \\
\hline Large body movements/min & 0.29 & 0.26 & $0.03-1.01$ \\
\hline Total body movements/min & 0.76 & 0.49 & $0.20-1.93$ \\
\hline \multicolumn{4}{|l|}{ Indeterminate sleep $(n=6)$} \\
\hline Arousal duration (s/min) & 20.64 & 16.47 & $0-46.67$ \\
\hline Arousal number/min & 0.47 & 0.35 & $0-1.00$ \\
\hline $\mathrm{REM} / \mathrm{min}$ & 0.21 & 0.35 & $0-0.86$ \\
\hline Head movements/min & 0.02 & 0.06 & $0-0.14$ \\
\hline Face movements/min & 0.17 & 0.23 & $0-0.57$ \\
\hline Small body movements/min & 0.07 & 0.07 & $0-0.14$ \\
\hline Large body movements/min & 0.96 & 0.84 & $0-2.00$ \\
\hline Total body movements/min & 1.21 & 1.00 & $0-2.14$ \\
\hline \multicolumn{4}{|l|}{ All sleep states } \\
\hline Latency to state $1(\min )(n=12)$ & 33.75 & 22.25 & $0-63.00$ \\
\hline Efficiency $(n=16)$ & 0.99 & 0.02 & $0.96-1.00$ \\
\hline Maintenance $(n=16)$ & 0.99 & 0.02 & $0.96-1.00$ \\
\hline Cycle length $(\min )(n=16)$ & 13.13 & 10.87 & $4.00-43.00$ \\
\hline
\end{tabular}

Body movements. State-specific decreases in small and large body movements were noted with increasing postconceptional age. Such changes were noted only during quiet sleep, with an $r$ of $-0.53(p<0.001)$ for large body movements and an $r$ of $-0.38(p<0.02)$ for small body movements.

In contrast, head and facial movements showed a statespecific increase in number with maturation during active sleep, with a positive $r$ of $0.44(p<0.005)$ for head movements and an $r$ of $0.33(p<0.05)$ for face movements, and during indeterminate sleep with a positive $r$ of $0.39(p<0.05)$ for head movements and a positive $r$ of $0.50(p<0.005)$ for facial movements.

\section{DISCUSSION}

Comparisons were made between each of eight phasic and 14 continuity measures with increasing postconceptional ages from 28 to $35 \mathrm{wk}$ in 43 healthy preterm neonates. Increasing numbers and durations of arousals were noted during continuous EEG. This finding should be considered in the context of our previously reported findings of decreased arousal numbers and durations during quiet sleep in preterm infants when they reach postconceptional term ages (20). Neuronal mechanisms responsible for arousal appear to mature early during
Table 5. Descriptive statistics for postconceptional ages 32 to $35 \mathrm{wk}$

\begin{tabular}{cccc}
\hline Variable & Mean & SD & Range \\
\hline
\end{tabular}

Continuous sleep $(n=27)$

Postconceptional age (wk)

Arousal duration (s/min)

Arousal number/min

$\mathrm{REM} / \mathrm{min}$

Head movements/min

Face movements/min

Small body movements/min

Large body movements/min

Total body movements/min

Discontinuous sleep $(n=27)$

Arousal duration (s/min)

Arousal number/min

$\mathrm{REM} / \mathrm{min}$

Head movements/min

Face movements/min

Small body movements/min

Large body movements/min

Total body movements/min

Indeterminate sleep $(n=25)$

Arousal duration (s/min)

Arousal number/min

$\mathrm{REM} / \mathrm{min}$

Head movements/min

Face movements/min

Small body movements/min

Large body movements/min

Total body movements/min

All sleep states

Latency to state $1(\mathrm{~min})(n=27)$

Efficiency $(n=27)$

Maintenance $(n=27)$

Cycle length $(\min )(n=23)$

$\begin{array}{rrc}33.50 & 1.00 & 32.00-35.00 \\ 3.17 & 1.99 & 0-6.65 \\ 0.22 & 0.14 & 0-0.48 \\ 2.08 & 1.51 & 0.53-7.24 \\ 0.13 & 0.09 & 0-0.45 \\ 0.69 & 0.32 & 0.08-1.24 \\ 0.15 & 0.16 & 0-0.69 \\ 0.28 & 0.11 & 0.13-0.60 \\ 1.25 & 0.45 & 0.53-2.17 \\ & & \\ 0.30 & 0.41 & 0-1.26 \\ 0.02 & 0.03 & 0-0.11 \\ 0.01 & 0.03 & 0-0.17 \\ 0.05 & 0.06 & 0-0.17 \\ 0.46 & 0.43 & 0-1.44 \\ 0.06 & 0.06 & 0-0.23 \\ 0.11 & 0.12 & 0-0.58 \\ 0.68 & 0.51 & 0-1.83 \\ & & \\ 25.77 & 10.44 & 0-43.53 \\ 0.73 & 0.34 & 0-1.14 \\ 0.63 & 0.73 & 0-2.77 \\ 0.26 & 0.24 & 0-1.00 \\ 0.48 & 0.41 & 0-1.71 \\ 0.14 & 0.22 & 0-1.00 \\ 0.87 & 0.41 & 0-2.00 \\ 1.75 & 0.71 & 0.65-4.00 \\ & & \\ 12.59 & 15.45 & 0-58.00 \\ 0.87 & 0.10 & 0.71-1.00 \\ 0.88 & 0.10 & 0.71-1.00 \\ 31.57 & 23.86 & 6.00-92.00 \\ & & \end{array}$

gestation and are already segregated to the continuous EEG segments before $35 \mathrm{wk}$ postconceptional age. Major maturational changes in the reticular activating system that subserve arousals seem to occur during continuous rather than during discontinuous EEG states in the preterm neonate.

Changes in sleep maintenance and efficiency measures with increasing postconceptional age have not been previously reported for preterm infants. Decreasing trends

Table 6. Spearman correlations of phasic measures with postconceptional age by EEG sleep state in preterm infants

\begin{tabular}{|c|c|c|c|c|c|c|}
\hline & \multicolumn{2}{|c|}{$\begin{array}{c}\text { Continuous } \\
\text { sleep } \\
(n=43) \\
\end{array}$} & \multicolumn{2}{|c|}{$\begin{array}{c}\text { Discontinuous } \\
\text { sleep } \\
(n=43) \\
\end{array}$} & \multicolumn{2}{|c|}{$\begin{array}{c}\text { Indeterminate } \\
\text { sleep } \\
(n=31)\end{array}$} \\
\hline & $r$ & $p$ & $r$ & $p$ & $r$ & $p$ \\
\hline \multicolumn{7}{|c|}{ Body movements } \\
\hline Head & 0.44 & $<0.005$ & & & 0.39 & $<0.05$ \\
\hline Face & 0.33 & $<0.05$ & & & 0.50 & $<0.005$ \\
\hline Small & & & -0.38 & 0.02 & & \\
\hline Large & & & -0.53 & 0.001 & & \\
\hline \multicolumn{7}{|l|}{ Arousals } \\
\hline Number & 0.69 & $<0.001$ & & & & \\
\hline Duration & 0.68 & $<0.001$ & & & & \\
\hline $\mathrm{REM} / \mathrm{min}$ & & & -0.39 & $<0.01$ & 0.54 & $<0.002$ \\
\hline
\end{tabular}


Table 7. Spearman correlations of continuity measures with postconceptional age

\begin{tabular}{lccc}
\hline & $r$ & $p$ & $n$ \\
\hline Latency & -0.42 & $<0.01$ & 39 \\
Efficiency & -0.72 & $<0.001$ & 43 \\
Maintenance & -0.72 & $<0.001$ & 43 \\
Cycle length (state 2 to state 2) & 0.59 & $<0.001$ & 39 \\
\hline
\end{tabular}

of these two polysomnographic variables reflect the neonate's improved ability to arouse, as previously discussed in terms of arousal numbers and durations. An improved ability to maintain arousal may secondarily result in longer periods of active sleep, which also increase with maturation (1).

Longer sleep-cycle periods and shorter sleep latencies before active sleep were also noted with increasing postconceptional age. This culminates in longer sleep cycles in preterm infants by a postconceptional term age than in the full-term infants (18). Lengthening of the sleep cycle is apparently occurring during prematurity, before the neonates reach postconceptional term ages. Others have also confirmed these sequential changes in EEG continuity and discontinuity with increasing postconceptional age $(9-12,16)$.

Motility during neonatal sleep has been reported (14 $18,21-24)$. Decreases in the numbers of small and large body movements during discontinuous EEG reflect the early maturation of certain state-specific motor behaviors. By a postconceptional term age, fewer large body movements are noted during quiet sleep age as compared to full-term infants (20). Kohyama and Iwakawa (14) also reported a decrease in gross body movements with increasing age (i.e. 33-184 postconceptional $\mathrm{wk}$ ), however, these were older infants and movements were not identified by EEG sleep state. Corticospinal pathways that mediate these motor behaviors may therefore be affected by specific states of arousal.

Increases in head and facial movements with maturation only during active and indeterminate sleep follow a different maturational trend, possibly reflecting an emerging postural reflex. Increased numbers of head movements in the older preterm infant may facilitate the appearance of the tonic neck reflex, which is a postural reflex only elicitable on examination after 32 wk EGA (19).

Although Curzi-Dascalova (17) found a predominance of body movements during active sleep in the preterm neonate, this author reported no decreasing trends with maturation. However, the small population sizes in that study during each postconceptional age range may, in part, explain the discrepancies with our findings. Others, however, also reported decreased body movement counts with increasing age $(14,15)$. What is more, specific aspects of body movements during active and quiet sleep have also been previously documented (5).

We have reaffirmed findings regarding REM by CurziDascalova et al. $(17,18)$, who reported an association between REM occurrences and specific sleep states. They reported that all infants after 31 wk postconceptional age with more than 5 min of "stable active sleep" were associated with the presence of REM. Our more strict definition of EEG continuity allowed us to reach the same conclusion in an inverse manner for discontinuous EEG segments; significant decreases in REM were noted during discontinuous EEG state (i.e. quiet sleep). Older preterm infants (i.e. $>33 \mathrm{wk}$ ) in our study also demonstrated more REM during indeterminate or transitional state segments, as reported by Kodyama et al. (15).

Normative data such as those presented in our report help define specific neurophysiologic behaviors during sleep in the healthy, asymptomatic preterm neonate. Such data can be compared with sleep behavior in neonates with suspected brain disorders. Several reports have already described altered movement patterns and sleep-state disturbances in full-term neonates with either structural brain lesions or encephalopathies $(6,21-26)$.

Conclusions reached from these exploratory analyses are only tentative. There is a comparison-wise error rate; each sleep measure was correlated separately with increased postconceptional age. State organization, on the other hand, is more accurately defined by interactions among multiple polysomnographic parameters (i.e. EEG, somatotopic, autonomic, and arousal measures) that have predictable temporal relationships (17). We are therefore examining multiple neonatal sleep measures with appropriate statistical algorithms that will analyze physiologic phenomena that may not be normally distributed and change continuously during varying levels of arousal (27).

\section{REFERENCES}

1. Pope JE, Werner SS, Bickford EG 1992 Atlas of Neonatal Electroencephalography, 2nd Ed. Raven Press, New York

2. Dreyfus-Brisac C 1970 Ontogenesis of sleep in human prematures after 32 weeks of conceptional age. Dev Psychobiol 3:91-121

3. Karch D, Rothe R, Jurisch R, Heldt-Hildebrandt R, Lubbesmeier A, Lemburg P 1982 Behavioral changes and bioelectric brain maturation of preterm and full-term newborn infants: a polygraphic study. Dev Med Child Neurol 24:30-47

4. Lombroso CT 1985 Neonatal polygraphy in full-term and premature infants: a review of normal and abnormal findings. J Clin Neurophysiol 2:105-155

5. Hakamada S, Watanabe K, Hara K, Miyazaki S 1981 Development of motor behavior during sleep in newborn infants. Brain Dev 3:345-350

6. Hakamada S, Watanabe K, Hara K, Miyazaki S, Kumagai T 1982 Body movements during sleep in full-term newborn infants. Brain Dev 4:51-55

7. Peirano P, Curzi-Dascalova L, Korn G 1986 Influence of sleep state and age on body motility in normal premature and full-term neonates. Neuropediatrics $17: 186-190$

8. Curzi-Dascalova L, Peirano P, Vicente G 1985 Body motility according to sleep states in normal newborn infants: a preliminary study. In: Jones CT, Nathaniels PW (eds) The Physiological Development of the Fetus and Newborn. Academic Press, London, pp 817-820

9. Connell JA, Oozeer R, Dubowitz V 1987 Continuous 4-channel EEG monitoring: a guide to interpretation, with normal values, in preterm infants. Neuropediatrics 18:138-145

10. Eyre JA, Nanei S, Wilkinson AR 1988 Quantification of normal neonatal EEGs with gestation from continuous five-day recordings. Develop Med Child Neurol 30:594-607

11. Anderson CM, Torres F, Faoro A 1985 The EEG of the early premature. Electroencephalogr Clin Neurophysiol 60:95-105

12. Thornberg E, Thiringer K 1990 Normal pattern of the cerebral function monitor trace in term and preterm neonates. Acta Paediatr Scand 79:20-25

13. Van Sweden B, Koenderink M, Windau G, Van de Bor M, Van Bel F, Van Dijk JG, Wauquier A 1991 Long-term EEG monitoring in the early premature: developmental and chronobiological aspects. Electroencephalogr Clin Neurophysiol 79:94-100

14. Kohyama J, Iwakawa Y 1990 Developmental changes in phasic sleep param- 
eters as reflections of the brain-stem maturation: polysomnographical examinations of infants, including premature neonates. Electroencephalogr Clin Neurophysiol 76:325-330

15. Kohyama J, Iwakawa Y 1991 Interrelationships between rapid eye and body movements during sleep: polysomnographic examinations of infants including premature neonates. Electroencephalogr Clin Neurophysiol 79:277-280

16. Goto K, Wakayama K, Sonoda $H$, Ogawa $T 1992$ Sequential changes in electroencephalogram continuity in very premature infants. Electroencephalogr Clin Neurophysiol 82:197-202

17. Curzi-Dascalova L 1992 Physiological correlates of sleep development in premature and full-term neonates. Neurophysiol Clin 22:151-166

18. Curzi-Dascalova L, Peirano P, Morel-Kahn F 1988 Development of sleep states in normal premature and full-term newborns. Dev Psychobiol 21:431-444

19. Dubowitz C, Dubowitz V 1981 The Neurological Assessment of the Preterm and Fullterm Newborn Infants. Clinics in Developmental Medicine, No. 79. Spastics International Medical Publications, JB Lippincott, Philadelphia

20. Scher MS, Steppe DA, Dahl RE, Asthana S, Guthrie RD 1992 Comparison of EEG-sleep measures in healthy full-term and preterm infants at matched conceptional ages. Sleep 15:442-448
21. Touwen BCL 1990 Variability and sterotypy of spontaneous motility as a predictor of neurological development of preterm infants. Dev Med Child Neurol 32:501-508

22. Erkinjuntti M 1988 Body movements during sleep in healthy and neurologically damaged infants. Early Hum Dev 16:283-292

23. Ferrari F, Cioni G, Prechtl HFR 1990 Qualitative changes of general movements in preterm infants with brain lesions. Early Hum Dev 23:193-231

24. Prechtl HFR 1990 Qualitative changes of spontaneous movements in fetus and preterm infant are a marker of neurological dysfunction. Early Hum Dev 23:151-158

25. Scher MS, Richardson GA, Coble PA, Day NL, Stoffer DS 1988 The effects of prenatal alcohol and marijuana exposure: disturbances in neonatal sleep cycling and arousal. Pediatr Res 24:101-105

26. Scher MS, Richardson GA, Salerno DG, Day NL, Guthrie RD 1992 Sleep architecture and continuity measures in neonates with chronic lung disease. Sleep 15:195-201

27. Scher MS, Dokianakis SG, Steppe DA, Banks DL, Sun M, Guthrie RD, Sclabassi RJ 1993 Relationships of multiple sleep measures with degrees of EEG discontinuity in preterm neonates. Sleep Res 22:67(abstr)

\section{Announcement}

\section{Annual Meetings}

The American Pediatric Society, The Society for Pediatric Research, and The Ambulatory Pediatric Association will hold their annual meetings May 8-11, 1995, at the San Diego Convention Center, San Diego, CA.

For further information, contact: APS/SPR Association Headquarters, 141 Northwest Point Blvd., P.O. Box 675, Elk Grove Village, IL 60009-0675, (708) 427-0205, Fax: (708) 427-1305 or Ambulatory Pediatric Association, 6728 Old McLean Village, McLean, VA 22101, (703) 556-9222, Fax: (703) 556-8729. 